The average position of the first maximum in a sample of geometric random variables 


\title{
Tail Bounds for the Wiener Index of Random Trees
}

\author{
Tämur Ali Khan and Ralph Neiningerf \\ Department for Mathematics and Computer Science, J.W. Goethe-University Frankfurt, \\ 60054 Frankfurt a. M., Germany
}

received $17 \mathrm{Feb} 2007$, revised $19^{\text {th }}$ January 2008 , accepted .

Upper and lower bounds for the tail probabilities of the Wiener index of random binary search trees are given. For upper bounds the moment generating function of the vector of Wiener index and internal path length is estimated. For the lower bounds a tree class with sufficiently large probability and atypically large Wiener index is constructed. The methods are also applicable to related random search trees.

\section{Contents}

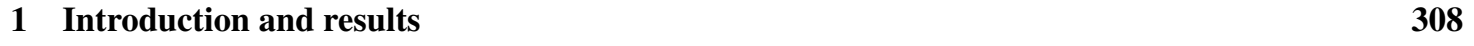

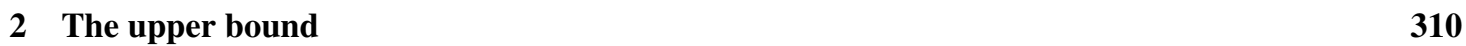

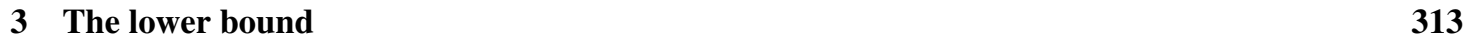

\section{Introduction and results}

The Wiener index of a connected graph is the sum of the distances between all unordered pairs of vertices of the graph. The distance between two vertices is defined as the minimum number of edges connecting them. The index was introduced by the chemist Wiener in 1947, in order to study relations between organic compounds and the index of their molecular graphs. For trees the Wiener index has been studied by discrete mathematicians and chemists, cf. the survey of (DEG01).

For random tree models comparatively little is known about the Wiener index. (EMMS94) studied the average Wiener index of simply generated families of trees and showed that the average is asymptotically $K n^{5 / 2}$, where $K$ is a constant depending on the simply generated family and $n \rightarrow \infty$ denotes the number of nodes. For some of these families (ordinary rooted trees, rooted labeled trees and rooted binary trees) they also gave exact formulæ for the expected Wiener index. (Jan03) proved a limit law for the Wiener index of these tree classes and identified the limit as a functional of the Brownian excursion. (FJ07) studied the right tail of this limit. Average Wiener indices of some other tree classes were computed by (Wag06; Wag07).

\footnotetext{
${ }^{\dagger}$ Supported by an Emmy Noether Fellowship of the Deutsche Forschungsgemeinschaft. 
In this paper we present tail bounds for the Wiener index $W_{n}$ of random binary search trees with $n$ internal nodes. The average Wiener index of random binary search trees was derived in ( $\mathrm{HN} 02)$,

$$
\mathbb{E} W_{n}=2 n^{2} H_{n}-6 n^{2}+8 n H_{n}-10 n+6 H_{n},
$$

where $H_{n}$ denotes the harmonic number $H_{n}=\sum_{j=1}^{n} 1 / j$. In (Nei02) the Wiener index of random binary search trees and random recursive trees was studied with respect to limit laws. By setting up a bivariate distributional recurrence for the Wiener index and the internal path length techniques from the contraction method could be used. For the tail bounds of the present paper we also use this recursive description: We denote by $\left(W_{n}, P_{n}\right)$ the vector of Wiener index and internal path length of the random binary search tree with $n$ internal nodes, and by $I_{n}$ and $J_{n}=n-1-I_{n}$ the cardinalities of the left and right subtree of the root. Then, $I_{n}$ and $J_{n}$ are uniformly distributed on $\{0, \ldots, n-1\}$. We have the recurrence,

$$
\left(\begin{array}{c}
W_{n} \\
P_{n}
\end{array}\right) \stackrel{d}{=}\left[\begin{array}{cc}
1 & n-I_{n} \\
0 & 1
\end{array}\right]\left(\begin{array}{c}
W_{I_{n}} \\
P_{I_{n}}
\end{array}\right)+\left[\begin{array}{cc}
1 & n-J_{n} \\
0 & 1
\end{array}\right]\left(\begin{array}{c}
W_{J_{n}}^{\prime} \\
P_{J_{n}}^{\prime}
\end{array}\right)+\left(\begin{array}{c}
2 I_{n} J_{n}+n-1 \\
n-1
\end{array}\right),
$$

where $\left(W_{i}, P_{i}\right),\left(W_{j}^{\prime}, P_{j}^{\prime}\right), 0 \leq i, j \leq n-1, I_{n}$ are independent and $\mathcal{L}\left(W_{j}^{\prime}, P_{j}^{\prime}\right)=\mathcal{L}\left(W_{j}, P_{j}\right)$. For the rescaled quantities $\mathbf{Y}_{0}=(0,0)$ and

$$
\mathbf{Y}_{n}=\left(\frac{W_{n}-\mathbb{E} W_{n}}{n^{2}}, \frac{P_{n}-\mathbb{E} P_{n}}{n}\right), \quad n \geq 1,
$$

a bivariate limit law and convergence of the covariance matrix has been shown, see (Nei02).

Here, we present the following tail bounds:

Theorem 1.1 Let $L_{0} \doteq 5.0177$ be the largest root of $e^{L}=6 L^{2}$ and $c=\left(L_{0}-1\right) /\left(24 L_{0}^{2}\right) \doteq 0.0066$. Then we have for every $t>0$ and every $n \geq 0$

$$
\mathbb{P}\left(\frac{W_{n}-\mathbb{E} W_{n}}{n^{2}} \geq t\right) \leq \begin{cases}\exp \left(-t^{2} / 36\right), & \text { for } 0 \leq t \leq 8.82 \\ \exp \left(-t^{2} / 96\right), & \text { for } 8.82<t \leq 48 L_{0} \\ \exp \left(-c t^{2}\right), & \text { for } 48 L_{0}<t \leq 24 L_{0}^{2} \\ \exp (-t(\log t-\log (4 e)), & \text { for } 24 L_{0}^{2}<t\end{cases}
$$

The same bound applies to the left tail.

We denote iterated logarithms by $\log ^{(k)} n$, i.e., $\log ^{(1)} n:=\log n$ and $\log ^{(k+1)} n:=\log \left(\log { }^{(k)} n\right)$ for $k \geq 1$.

Theorem 1.2 For all $t>0$ and all $n \geq 0$ we have

$$
\mathbb{P}\left(\left|W_{n}-\mathbb{E} W_{n}\right| \geq t \mathbb{E} W_{n}\right) \leq \exp \left(-2 t \log n\left(\log ^{(2)} n+\log t-\log (2 e)+o(1)\right)\right),
$$

where the o(1) is with respect to $n \rightarrow \infty$ and can also explicitly be bounded.

Furthermore we have a lower bound on the tail probabilities of $W_{n}$ :

Theorem 1.3 For all fixed $t>0$ and all sufficiently large $n$ we have

$$
\mathbb{P}\left(W_{n}-\mathbb{E} W_{n}>t \mathbb{E} W_{n}\right) \geq \exp \left(-8 t \log n\left(\log ^{(2)} n+O\left(\log ^{(3)} n\right)\right)\right) .
$$


To derive upper tail bounds in Section 2 we estimate the moment generating function $\mathbb{E} \exp \left\langle\mathbf{s}, \mathbf{Y}_{n}\right\rangle$, $\mathbf{s} \in \mathbb{R}^{2}$, from above, see Proposition 2.1. so that tail bounds can be obtained by Chernoff's bounding technique. The bounds for $\mathbb{E} \exp \left\langle\mathbf{s}, \mathbf{Y}_{n}\right\rangle$ are proved by induction on $n$ using recurrence 2 for the induction step. For this, we extend the analysis of the tails of the Quicksort complexity as given in (Rös91) and refined in (FJ02) to our two-dimensional setting. Note that the second component of $\mathbf{Y}_{n}$ is distributed as the normalized number of key comparisons used by Quicksort.

Another approach to tail bounds is via the method of bounded differences. A Doob martingale on $W_{n}$ can be defined via an appropriate filtration and its martingale differences can be estimated. We extended earlier analysis of (MH96) for the Quicksort complexity to the Wiener index but do not discuss this here since the resulting bounds we obtained are not tighter than the ones found by the approach presented. However, details of the application of the method of bounded differences to our problem can be found in the dissertation of (AK06), where also proofs that we omit subsequently are worked out.

In Section 3 we prove Theorem 1.3 . For this we construct a class of binary search trees having atypically large Wiener indices and show that the random binary search tree is in that class with sufficiently large probability. This construction also builds upon the analysis of (MH96) for lower tail bounds for $P_{n}$.

The methods used are applicable to related random search trees such as random (point) quad trees or random $m$-ary search trees and depend on a precise expansion of the average Wiener index of the tree.

\section{The upper bound}

Our tail bounds in Theorem 1.1 are based on the following estimate.

Proposition 2.1 Let $L_{0}$ be as in Theorem 1.1 and $\mathbf{s} \in \mathbb{R}^{2}$. Then for every $n \geq 1$

$$
\mathbb{E} \exp \left\langle\mathbf{s}, \mathbf{Y}_{n}\right\rangle \leq \begin{cases}\exp \left(9\|\mathbf{s}\|^{2}\right), & \text { for } 0 \leq\|\mathbf{s}\| \leq 0.49 \\ \exp \left(24\|\mathbf{s}\|^{2}\right), & \text { for } 0.49<\|\mathbf{s}\| \leq L_{0} \\ \exp \left(4 e^{\|\mathbf{s}\|}\right), & \text { for } L_{0}<\|\mathbf{s}\| .\end{cases}
$$

To scetch the proof we introduce the following notation: We set $w_{n}=\mathbb{E} W_{n}$ and $p_{n}=\mathbb{E} P_{n}$. Furthermore, for $1 \leq i \leq n-1$ and $j=j(i)=n-i-1$ we denote

$$
\begin{aligned}
a_{n}^{(1)}(i) & =\left[\begin{array}{cc}
(i / n)^{2} & i(n-i) / n^{2} \\
0 & i / n
\end{array}\right], \\
a_{n}^{(2)}(i) & =a_{n}^{(1)}(j), \\
C_{n}^{(1)}(i) & =\frac{1}{n^{2}}\left(w_{i}+(n-i) p_{i}+w_{j}+(n-j) p_{j}-w_{n}+2 i j+n-1\right), \\
C_{n}^{(2)}(i) & =\frac{1}{n}\left(p_{i}+p_{j}-p_{n}+n-1\right)
\end{aligned}
$$

and $\mathbf{C}_{n}(i)=\left(C_{n}^{(1)}(i), C_{n}^{(2)}(i)\right)$. With this notation the recurrence for $\mathbf{Y}_{n}$ induced by recurrence (2) reads

$$
\mathbf{Y}_{n} \stackrel{d}{=} A_{n}^{(1)} \mathbf{Y}_{I_{n}}+A_{n}^{(2)} \mathbf{Y}_{J_{n}}^{\prime}+\mathbf{b}_{n}, \quad n \geq 1
$$

with

$$
\left(A_{n}^{(1)}, A_{n}^{(2)}, \mathbf{b}_{n}\right)=\left(a_{n}^{(1)}\left(I_{n}\right), a_{n}^{(2)}\left(I_{n}\right), \mathbf{C}_{n}\left(I_{n}\right)\right)
$$


where $\mathbf{Y}_{i}, \mathbf{Y}_{j}^{\prime}, 0 \leq i, j \leq n-1, I_{n}$ are independent and $\mathcal{L}\left(\mathbf{Y}_{j}^{\prime}\right)=\mathcal{L}\left(\mathbf{Y}_{j}\right)$.

We collect some useful but technical estimates. We denote by $A^{T}$ the transpose of a matrix $A$ and set $\|A\|_{\text {op }}:=\sup _{\|x\|=1}\|A x\|$.

Lemma 2.2 Let $U$ be uniformly distributed on $[0,1]$ and couple $I_{n}, n \geq 1$, to $U$ by setting $I_{n}=\lfloor U n\rfloor$. Then we have for all $n \geq 1$,

$$
\left\|A_{n}^{(1) T} A_{n}^{(1)}\right\|_{\mathrm{op}}+\left\|A_{n}^{(2) T} A_{n}^{(2)}\right\|_{\mathrm{op}}-1<-U(1-U) .
$$

Lemma 2.3 We have

$$
\sup _{n \geq 0} \max _{1 \leq i \leq n-1}\left\|\mathbf{C}_{n}(i)\right\|=1
$$

Proof of Proposition 2.1; The assertion follows from the next result by choosing $L=\|\mathbf{s}\|$ : For every $L>0$, denote

$$
K_{L}= \begin{cases}9, & \text { for } L \leq 0.49 \\ 24, & \text { for } 0.49<L \leq L_{0} \\ 4 e^{L} / L^{2}, & \text { for } L_{0}<L\end{cases}
$$

Then

$$
\mathbb{E} \exp \left\langle\mathbf{s}, \mathbf{Y}_{n}\right\rangle \leq \exp \left(K_{L}\|\mathbf{s}\|^{2}\right)
$$

for every $\|\mathbf{s}\| \leq L, n \geq 0$. This will be proved by induction on $n$. For $n=0$ we have $\mathbf{Y}_{0}=(0,0)$ and the assertion is true. Assume the assertion is true for some $L>0,\|\mathbf{s}\| \leq L$ and every $0 \leq i \leq n-1$. Then, conditioning on $I_{n}=\lfloor U n\rfloor=i$ and using the distributional recurrence 3 we obtain for $j=n-i-1$ and $\|\mathbf{s}\| \leq L$,

$$
\begin{aligned}
\mathbb{E} \exp \left\langle\mathbf{s}, \mathbf{Y}_{n}\right\rangle & =\frac{1}{n} \sum_{i=0}^{n-1} \exp \left\langle\mathbf{s}, \mathbf{C}_{n}(i)\right\rangle \mathbb{E} \exp \left\langle\mathbf{s}, a_{n}^{(1)}(i) \mathbf{Y}_{i}\right\rangle \mathbb{E} \exp \left\langle\mathbf{s}, a_{n}^{(2)}(i) \mathbf{Y}_{j}\right\rangle \\
& \leq \frac{1}{n} \sum_{i=0}^{n-1} \exp \left\langle\mathbf{s}, \mathbf{C}_{n}(i)\right\rangle \exp \left(K_{L}\left\|a_{n}^{(1)}(i)^{T} \mathbf{s}\right\|^{2}+K_{L}\left\|a_{n}^{(2)}(i)^{T} \mathbf{s}\right\|^{2}\right) \\
& \leq \frac{1}{n} \sum_{i=0}^{n-1} \exp \left(\left\langle\mathbf{s}, \mathbf{C}_{n}(i)\right\rangle+K_{L}\|\mathbf{s}\|^{2} \sum_{r=1}^{2}\left\|a_{n}^{(r)}(i)^{T} a_{n}^{(r)}(i)\right\|_{\mathrm{op}}\right) \\
& =\mathbb{E} \exp \left(\left\langle\mathbf{s}, \mathbf{b}_{n}\right\rangle+K_{L}\|\mathbf{s}\|^{2} \sum_{r=1}^{2}\left\|A_{n}^{(r) T} A_{n}^{(r)}\right\|_{\mathrm{op}}\right) \\
& \leq \mathbb{E} \exp \left(\left\langle\mathbf{s}, \mathbf{b}_{n}\right\rangle+K_{L}\|\mathbf{s}\|^{2}(1-U(1-U))\right) \\
& =\mathbb{E} \exp \left(\left\langle\mathbf{s}, \mathbf{b}_{n}\right\rangle-K_{L}\|\mathbf{s}\|^{2} U(1-U)\right) \exp \left(K_{L}\|\mathbf{s}\|^{2}\right) .
\end{aligned}
$$

For (5) we applied the induction hypothesis, using

$$
\left\|a_{n}^{(r)}(i)^{T} \mathbf{s}\right\| \leq\left\|a_{n}^{(r)}(i)^{T} a_{n}^{(r)}(i)\right\|_{\text {op }}^{1 / 2}\|\mathbf{s}\| \leq\|\mathbf{s}\| \leq L
$$


since $\left\|a_{n}^{(r)}(i)^{T} a_{n}^{(r)}(i)\right\|_{\mathrm{op}} \leq 1$ for $r=1,2,0 \leq i \leq n-1$, and for 6 we applied Lemma 2.2. Hence the proof is completed by showing that

$$
\sup _{n \geq 0} \mathbb{E} \exp \left(\left\langle\mathbf{s}, \mathbf{b}_{n}\right\rangle-K_{L}\|\mathbf{s}\|^{2} U(1-U)\right) \leq 1 .
$$

We consider the cases $L \leq 0.49$ and $L \geq 0.49$ separately.

$L \leq 0.49$ : The Cauchy-Schwarz inequality yields

$$
\begin{aligned}
& \mathbb{E} \exp \left(\left\langle\mathbf{s}, \mathbf{b}_{n}\right\rangle-K_{L}\|\mathbf{s}\|^{2} U(1-U)\right) \\
& \leq \mathbb{E} \exp \left(2\left\langle\mathbf{s}, \mathbf{b}_{n}\right\rangle\right)^{1 / 2} \mathbb{E} \exp \left(-2 K_{L}\|\mathbf{s}\|^{2} U(1-U)\right)^{1 / 2},
\end{aligned}
$$

thus it suffices to prove

$$
\mathbb{E} \exp \left(2\left\langle\mathbf{s}, \mathbf{b}_{n}\right\rangle\right) \mathbb{E} \exp \left(-2 K_{L}\|\mathbf{s}\|^{2} U(1-U)\right) \leq 1 .
$$

With $\left\|\mathbf{b}_{n}\right\|_{\infty} \leq 1$ by Lemma 2.3 and $\mathbb{E}\left\langle\mathbf{s}, \mathbf{b}_{n}\right\rangle=0$ we obtain

$$
\begin{aligned}
\mathbb{E} \exp \left(2\left\langle\mathbf{s}, \mathbf{b}_{n}\right\rangle\right) & =\mathbb{E}\left(1+2\left\langle\mathbf{s}, \mathbf{b}_{n}\right\rangle+\sum_{k=2}^{\infty} \frac{\left(2\left\langle\mathbf{s}, \mathbf{b}_{n}\right\rangle\right)^{k}}{k !}\right) \\
& =1+\mathbb{E}\left\langle\mathbf{s}, \mathbf{b}_{n}\right\rangle^{2} \sum_{k=2}^{\infty} \frac{2^{k}\left\langle\mathbf{s}, \mathbf{b}_{n}\right\rangle^{k-2}}{k !} \\
& \leq 1+\|\mathbf{s}\|^{2} \sum_{k=2}^{\infty} \frac{2^{k}(1 / 2)^{k-2}}{k !} \\
& =1+\|\mathbf{s}\|^{2} 4(e-2) .
\end{aligned}
$$

With $K_{L}=9$ we have

$$
\mathbb{E} \exp \left(-2 K_{L}\|\mathbf{s}\|^{2} U(1-U)\right) \leq 1-3\|\mathbf{s}\|^{2}+\frac{27}{5}\|\mathbf{s}\|^{4},
$$

using $\exp (-x) \leq 1-x+x^{2} / 2$ for $x \geq 0$. Furthermore, one easily checks that for $\|s\| \leq 0.49$ we have

$$
\left(1+\|\mathbf{s}\|^{2} 4(e-2)\right)\left(1-3\|\mathbf{s}\|^{2}+\frac{27}{5}\|\mathbf{s}\|^{4}\right) \leq 1 .
$$

Thus (7) and (8) yield that (4) is true for $\|\mathbf{s}\| \leq L \leq 0.49$ with $K_{L}=9$.

$L>0.49$ : Again, with $\left\|\mathbf{b}_{n}\right\|_{\infty} \leq 1$ we obtain

$$
\mathbb{E} \exp \left(\left\langle\mathbf{s}, \mathbf{b}_{n}\right\rangle-K_{L}\|\mathbf{s}\|^{2} U(1-U)\right) \leq \exp (\|\mathbf{s}\|) \mathbb{E} \exp \left(-K_{L}\|\mathbf{s}\|^{2} U(1-U)\right) .
$$

It is proved in Section 4 of (FJ01) that the right hand side of this inequality is smaller than 1 if $0.42 \leq$ $\|\mathbf{s}\| \leq 2$ and $K_{L}=24$, respectively if $2 \leq\|\mathbf{s}\| \leq L$ and $K_{L}=4 e^{L} / L^{2}$. Thus for $K_{L}=24 L^{2} \vee 4 e^{L} / L^{2}$ 
we have $\mathbb{E} \exp \left\langle\mathbf{s}, \mathbf{Y}_{n}\right\rangle \leq \exp \left(K_{L}\|\mathbf{s}\|^{2}\right)$, for every $\|\mathbf{s}\| \leq L, n \geq 0$. Since $24 L^{2} \geq 4 e^{L} / L^{2}$ for $L \leq L_{0}$ and $24 L^{2} \leq 4 e^{L} / L^{2}$ for $L>L_{0}$, this completes the proof.

Proof of Theorem 1.1; By standard arguments using Markov's inequality and Proposition 2.1, cf. the proof of Theorem 3.6 in (AKN04).

Proof of Theorem 1.2; Choose $t_{n}=t w_{n} / n^{2}=2 t \log n+O(1)$ in Theorem 1.1

\section{The lower bound}

In this section we prove Theorem 1.3 . The Wiener index of a binary search tree of order $n$ is rather large, if it has two subtrees which have a large distance from each other and which both have large sizes. Based on this observation we define for every fixed $t>0$ a class of binary search trees of order $n$. Every tree in that class has two subtrees with sufficiently large distance from each other and large sizes, such that conditioned on the event that the random binary search tree is in that class, the event $\left\{W_{n}-\mathbb{E} W_{n}>t \mathbb{E} W_{n}\right\}$ has probability tending to 1 , as $n \rightarrow \infty$. Moreover the probability that the random binary search tree is in that class is at least as large as the bound stated in Theorem 1.3 .

Proof of Theorem 1.3 ;o define the event $A$ that the random binary search tree is in the above mentioned class, we denote for fixed $t>0$

$$
\lambda:=\frac{\log ^{(3)} n}{\log ^{(2)} n}, \quad \kappa:=8+24 \lambda, \quad k:=\lfloor\kappa t \log n\rfloor, \quad s:=\left\lfloor\frac{\lambda n}{t \log n}\right\rfloor .
$$

We number nodes in the (complete) binary tree as follows. The root has number 1 and we count level by level from left to right, cf. figure 1. We denote by $S_{i}$ the size of the subtree rooted at node $i$ and set $S_{i}=0$ if node $i$ does not belong to the binary search tree. Note that by our count node $2^{m}+1$ is the second leftmost node on level $m$.

Let $A$ be the event that $S_{2}=\lfloor(n+1) / 2\rfloor$ and that $S_{2^{m}+1} \leq s-1$, for $2 \leq m \leq k$, see figure 1 . Thus under event $A$ we have $S_{3}=\lceil(n-3) / 2\rceil$ and $S_{2^{k}} \geq n / 2-(k-1) s$. Having two large subtrees this far away from each other will yield that $W_{n}$ is sufficiently large. First note that

$$
\begin{aligned}
\mathbb{P}(A) & \geq \frac{1}{n}\left(\frac{s}{(n+1) / 2}\right)^{k-1} \geq \frac{1}{n}\left(\frac{s}{n}\right)^{k-1} \\
& =\exp (-(k-1) \log (n / s)-\log n) \\
& \geq \exp \left(-8 t \log n\left(\log ^{(2)} n+O\left(\log ^{(3)} n\right)\right)\right) .
\end{aligned}
$$

From now on, we will assume w.l.o.g. that $n$ is even. The distance between two nodes in a tree is the number of edges connecting them. From this point of view the Wiener index of a tree can be calculated by counting how often each edge is passed when summing up all node distances. In our notation the incoming edge of node $i$ is passed $S_{i}\left(n-S_{i}\right)$ times. Thus

$$
W_{n}=\sum_{i \in \mathbb{N}} S_{i}\left(n-S_{i}\right)
$$




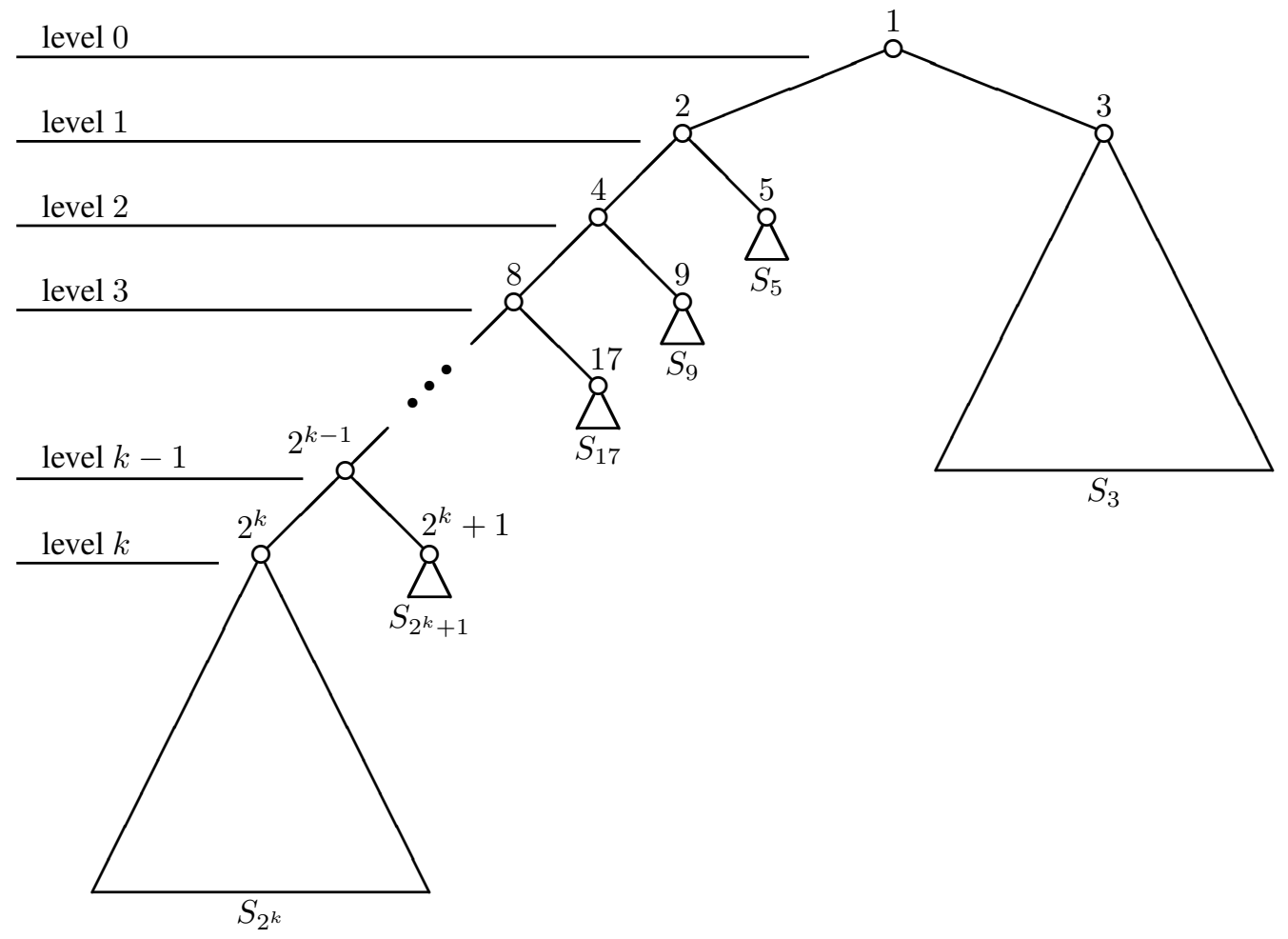

Fig. 1: Under event $A$ we have subtree sizes $S_{3}=\lceil(n-3) / 2\rceil$ and $S_{2^{m}+1} \leq s-1$, for $2 \leq m \leq k$, thus $S_{2^{k}} \geq n / 2-(k-1) s$.

where exactly $n-1$ of these summands are nonzero. We set

$$
W_{n}^{\prime}=\sum_{m=1}^{k} S_{2^{m}}\left(n-S_{2^{m}}\right)
$$

and $W_{n}^{\prime \prime}=W_{n}-W_{n}^{\prime}$ and estimate $W_{n}^{\prime}$ and $W_{n}^{\prime \prime}$ separately under event $A$. By construction, $W_{n}^{\prime}$ is the number of passings of the edges above the nodes $2^{m}, 1 \leq m \leq k$. For $\left(s_{2}, \ldots, s_{k}\right) \in M=\{1, \ldots, s\}^{k-1}$ let $A\left(s_{2}, \ldots, s_{k}\right)$ be the event that $S_{3}=\lceil(n+1) / 2\rceil$ and that $S_{2^{m}+1}=s_{m}-1$, for $2 \leq m \leq k$. Thus

$$
A=\bigcup_{\left(s_{2}, \ldots, s_{k}\right) \in M} A\left(s_{2}, \ldots, s_{k}\right) .
$$

We denote $\sigma_{1}=0$ and $\sigma_{m}=\sigma_{m-1}+s_{m}$ for $2 \leq m \leq k$. Then $(m-1) \leq \sigma_{m} \leq(m-1) s$ and under 
event $A\left(s_{2}, \ldots, s_{k}\right)$ we have

$$
\begin{aligned}
W_{n}^{\prime} & =\sum_{m=1}^{k}\left(\frac{n}{2}+\sigma_{m}\right)\left(\frac{n}{2}-\sigma_{m}\right)=\sum_{m=1}^{k}\left(\frac{n^{2}}{4}-\sigma_{m}^{2}\right) \geq \frac{k n^{2}}{4}-s^{2} \sum_{m=1}^{k}(m-1)^{2} \\
& \geq \frac{k n^{2}}{4}\left(1-\frac{4}{3} \frac{k^{2} s^{2}}{n^{2}}\right) \geq\left((1+3 \lambda) 2 t \log n-\frac{1}{4}\right) n^{2}\left(1-\frac{4}{3} \kappa^{2} \lambda^{2}\right) \\
& =2 t n^{2} \log n\left(1+3 \lambda-\frac{1}{8 t \log n}\right)\left(1-\frac{4}{3} \kappa^{2} \lambda^{2}\right) \\
& \geq 2 t(1+\lambda) n^{2} \log n,
\end{aligned}
$$

for sufficiently large $n$. For the last inequality in line $(10)$ we use

$$
\left(1+3 \lambda-\frac{1}{8 t \log n}\right)\left(1-\frac{4}{3} \kappa^{2} \lambda^{2}\right) \geq(1+2 \lambda)\left(1-\frac{4}{3} \kappa^{2} \lambda^{2}\right) \geq 1+\lambda
$$

for sufficiently large $n$.

In order to estimate $W_{n}^{\prime \prime}$ under event $A\left(s_{2}, \ldots, s_{k}\right)$ via Chebychev's inequality, we will use

$$
\begin{aligned}
\mathbb{E}\left(W_{n}^{\prime \prime} \mid A\left(s_{2}, \ldots, s_{k}\right)\right) \geq & w_{n / 2-1}+\left(\frac{n}{2}+1\right) p_{n / 2-1} \\
& +w_{n / 2-\sigma_{k}}+\left(\frac{n}{2}+\sigma_{k}\right) p_{n / 2-\sigma_{k}} \\
& +\sum_{m=2}^{k}\left(w_{s_{m}-1}+\left(n-s_{m}+1\right) p_{s_{m}-1}\right) .
\end{aligned}
$$

This inequality is valid, since the right hand side is the expected number of passings of all edges belonging to subtrees rooted at either node 3 (the summands in line $(11)$ ) or node $2^{k}$ (the summands in line $(12$ ) or node $2^{m}+1,2 \leq m \leq k$, (the summands in line (13). With $H_{\ell} \geq \log \ell$ we get for $\ell \leq n$

$$
\begin{aligned}
w_{\ell}+(n-\ell) p_{\ell} & \geq 2 \ell^{2} \log \ell-6 \ell^{2}+o\left(\ell^{2}\right)+(n-\ell)(2 \ell \log \ell-4 \ell) \\
& \geq n(2 \ell \log \ell-6 \ell+o(\ell)) .
\end{aligned}
$$

Thus

$$
\begin{aligned}
\mathbb{E}\left(W_{n}^{\prime \prime} \mid A\left(s_{2}, \ldots, s_{k}\right)\right) \geq & 2 n\left(\frac{n}{2}-1\right) \log \left(\frac{n}{2}-1\right)+2 n\left(\frac{n}{2}-\sigma_{k}\right) \log \left(\frac{n}{2}-\sigma_{k}\right) \\
& +\sum_{m=2}^{k} 2 n\left(s_{m}-1\right) \log \left(s_{m}-1\right)-6 n^{2}+o\left(n^{2}\right) \\
\geq & 2 n\left(n-\sigma_{k}-1\right) \log \left(\frac{n}{2}-\sigma_{k}\right)+2 n(k-1)(\hat{s}-1) \log (\hat{s}-1)-6 n^{2}+o\left(n^{2}\right),
\end{aligned}
$$

by convexity of $x \mapsto x \log x$, where $\hat{s}=1 /(k-1) \sum_{m=2}^{k} s_{m}$. With $\sigma_{k}=(k-1) \hat{s} \leq(k-1) s$ we have

$$
\begin{aligned}
\left(n-\sigma_{k}-1\right) \log \left(\frac{n}{2}-\sigma_{k}\right) & \geq(n-(k-1) \hat{s}-1)\left(\log n+\log \left(1-\frac{2(k-1) s}{n}\right)-\log 2\right) \\
& =n \log n-(\log 2) n-(k-1) \hat{s} \log n+o(n) .
\end{aligned}
$$


Together this yields

$$
\begin{aligned}
& \mathbb{E}\left(W_{n}^{\prime \prime} \mid A\left(s_{2}, \ldots, s_{k}\right)\right) \\
& \geq 2 n^{2} \log n-2 n(k-1)(\hat{s}-1) \log \left(\frac{n}{\hat{s}-1}\right)-(6+2 \log 2) n^{2}-2 n(k-1) \log n+o\left(n^{2}\right) \\
& \geq 2 n^{2} \log n-2 n(k-1)(s-1) \log \left(\frac{n}{s-1}\right)-(6+2 \log 2) n^{2}+o\left(n^{2}\right) \\
& =2 n^{2} \log n-2 \kappa \lambda n^{2} \log \left(\frac{t \log n}{\lambda}\right)-(6+2 \log 2) n^{2}+o\left(n^{2}\right) \\
& \geq 2 n^{2} \log n-(16+o(1)) n^{2} \log ^{(3)} n,
\end{aligned}
$$

for all sufficiently large $n$, where we use that $x \mapsto x \log (n / x)$ is increasing for $0<x<n / e$. Similarly to (13) we have

$$
\begin{aligned}
\operatorname{Var}\left(W_{n}^{\prime \prime} \mid A\left(s_{2}, \ldots, s_{k}\right)\right) & =\operatorname{Var}\left(W_{n / 2-1}+\left(\frac{n}{2}+1\right) P_{n / 2-1}\right) \\
& +\operatorname{Var}\left(W_{n / 2-\sigma_{k}}+\left(\frac{n}{2}+\sigma_{k}\right) P_{n / 2-\sigma_{k}}\right) \\
& +\sum_{m=2}^{k} \operatorname{Var}\left(W_{s_{m}-1}+\left(n-s_{m}+1\right) P_{s_{m}-1}\right) .
\end{aligned}
$$

For $\ell \leq n$,

$$
\begin{aligned}
\operatorname{Var}\left(W_{\ell}+(n-\ell) P_{\ell}\right) & =\operatorname{Var}\left(W_{\ell}\right)+(n-\ell)^{2} \operatorname{Var}\left(P_{\ell}\right)+2(n-\ell) \operatorname{Cov}\left(W_{\ell}, P_{\ell}\right) \\
& \leq O\left(\ell^{4}\right)+n^{2} O\left(\ell^{2}\right)+2 n O\left(\ell^{3}\right),
\end{aligned}
$$

since $\operatorname{Var}\left(W_{n}\right)=O\left(n^{4}\right)$ and $\operatorname{Cov}\left(W_{n}, P_{n}\right)=O\left(n^{3}\right)$, as shown in (Nei02), and $\operatorname{Var}\left(P_{n}\right)=O\left(n^{2}\right)$. Thus

$$
\operatorname{Var}\left(W_{n}^{\prime \prime} \mid A\left(s_{2}, \ldots, s_{k}\right)\right)=O\left(n^{4}\right)
$$

and hence by Chebychev's inequality

$$
\mathbb{P}\left(W_{n}^{\prime \prime} \geq 2 n^{2} \log n-17 n^{2} \log ^{(3)} n \mid A\left(s_{2}, \ldots, s_{k}\right)\right) \rightarrow 1 \quad \text { as } n \rightarrow \infty .
$$

This convergence is uniform over all $\left(s_{2}, \ldots, s_{k}\right) \in M$. For sufficiently large $n$,

$$
2 t(1+\lambda) n^{2} \log n+2 n^{2} \log n-17 n^{2} \log ^{(3)} n>(1+t) \mathbb{E} W_{n} .
$$


Using estimates (9), (10), (14) and (15) we get

$$
\begin{aligned}
& \mathbb{P}\left(W_{n}>(1+t) \mathbb{E} W_{n}\right) \\
& \geq \mathbb{P}\left(W_{n}>(1+t) \mathbb{E} W_{n} \mid A\right) \mathbb{P}(A) \\
& =\sum_{\left(s_{2}, \ldots, s_{k}\right) \in M} \mathbb{P}\left(W_{n}>(1+t) \mathbb{E} W_{n} \mid A\left(s_{2}, \ldots, s_{k}\right)\right) \mathbb{P}\left(A\left(s_{2}, \ldots, s_{k}\right)\right) \\
& \geq \sum_{\left(s_{2}, \ldots, s_{k}\right) \in M} \mathbb{P}\left(W_{n}^{\prime \prime}>2 n^{2} \log n-17 n^{2} \log ^{(3)} n \mid A\left(s_{2} \ldots, s_{k}\right)\right) \mathbb{P}\left(A\left(s_{2}, \ldots, s_{k}\right)\right) \\
& =(1+o(1)) \mathbb{P}(A) \\
& =\exp \left(-8 t \log n\left(\log ^{(2)} n+O\left(\log ^{(3)} n\right)\right)\right) .
\end{aligned}
$$

This completes the proof.

\section{References}

[AK06] Tämur Ali Khan. Concentration of Multivariate Random Recursive Sequences arising in the Analysis of Algorithms. Dissertation, J.W. Goethe-Universität Frankfurt a.M., 2006.

[AKN04] Tämur Ali Khan and Ralph Neininger. Probabilistic analysis for randomized game tree evaluation. In Mathematics and computer science. III, Trends Math., pages 163-174. Birkhäuser, Basel, 2004.

[DEG01] Andrey A. Dobrynin, Roger Entringer, and Ivan Gutman. Wiener index of trees: theory and applications. Acta Appl. Math., 66(3):211-249, 2001.

[EMMS94] R. C. Entringer, A. Meir, J. W. Moon, and L. A. Székely. The Wiener index of trees from certain families. Australas. J. Combin., 10:211-224, 1994.

[FJ01] James Allen Fill and Svante Janson. Approximating the limiting Quicksort distribution. Random Structures Algorithms, 19(3-4):376-406, 2001. Analysis of algorithms (Krynica Morska, 2000).

[FJ02] James Allen Fill and Svante Janson. Quicksort asymptotics. J. Algorithms, 44(1):4-28, 2002. Analysis of algorithms.

[FJ07] James Allen Fill and Svante Janson. Precise logarithmic asymptotics for the right tails of some limit random variables for random trees. Preprint, 2007.

[HN02] Hsien-Kuei Hwang and Ralph Neininger. Phase change of limit laws in the quicksort recurrence under varying toll functions. SIAM J. Comput., 31(6):1687-1722 (electronic), 2002.

[Jan03] Svante Janson. The Wiener index of simply generated random trees. Random Structures Algorithms, 22(4):337-358, 2003.

[MH96] C. J. H. McDiarmid and R. B. Hayward. Large deviations for Quicksort. J. Algorithms, 21(3):476-507, 1996. 
[Nei02] Ralph Neininger. The Wiener index of random trees. Combin. Probab. Comput., 11(6):587$597,2002$.

[Rös91] Uwe Rösler. A limit theorem for "Quicksort". RAIRO Inform. Théor. Appl., 25(1):85-100, 1991.

[Wag06] Stephan G. Wagner. A class of trees and its Wiener index. Acta Appl. Math., 91(2):119-132, 2006.

[Wag07] Stephan G. Wagner. On the average Wiener index of degree-restricted trees. Australas. J. Combin., 37:187-203, 2007. 